\title{
Origin of Three Fold Torsional Potential in Methylated 2-Hydroxypyridine
}

\author{
Ankit Kumar Srivastava ${ }^{1}$, Rajeev Kumar Sinha ${ }^{2}$, Swasti Saxena ${ }^{3}$ and Tapanendu Kundu ${ }^{4}$ \\ ${ }^{1}$ Department of Physics, Indian Institute of Technology Bombay, Mumbai, Maharastra, India \\ 400076. \\ ${ }^{2}$ Department of Atomic and Molecular Physics, Manipal University, Manipal, Karnataka, \\ India 576104 \\ ${ }^{3}$ Applied Physics Department, Sardar Vallabhbhai National Institute of Technology Surat, \\ Gujarat, India-395007 \\ ${ }^{4}$ Department of Physics, Indian Institute of Technology Bombay, Mumbai, Maharastra, India \\ 400076
}

\section{ARTICLE INFO}

\section{Keywords:}

Ab initio calculation, Hyperconjugation, Methyl torsion, REMPI.

\begin{abstract}
In this paper, the detailed study of methylated 2-hydroxypyridine molecule has been carried out using resonance enhanced multiphoton ionization (REMPI) spectroscopic technique. However, the theoretical investigations have been performed using ab initio calculations. The origin band of the molecules 4-methyl-2-hydroxypyridine (4M2HP) and 6-methyl-2-hydroxypyridine (6M2HP) was observed at $34987 \mathrm{~cm}-$ 1 and $35405 \mathrm{~cm}-1$ in their REMPI spectrum and the bands assigned as $\pi \pi^{*}$ transition state. The vibronic coupling of the $n \pi^{*}$ and $\pi \pi^{*}$ transition states took place in 4M2HP, thus some low intense bands near the origin band of the molecule were observed in the spectrum. However, no such kind of bands observed in 6M2HP. The $\pi^{*}-\sigma^{*}$ hyperconjugation is responsible for the conformational change of the methyl group in 4M2HP upon excitation $(\mathrm{S} 0 \rightarrow \mathrm{S} 1)$.
\end{abstract}

\section{Introduction}

The torsion motion can be defined as the twisting or pseudo rotation of a group of atoms or a part of molecule with respect to the whole molecule over a multiminima potential. Biphenyl is an example with torsion motion between the two rings. It has twisted angle of $420^{\circ}$ (Suzuki, 1959) while in solid state it is a planar molecule (Hargreaves \& Rizvi, 1962). The molecule has been studied in supersonic jet cooled condition by Im and Bernstein in 1988 (Im \& Bernstein, 1988). The electronic spectroscopy of the molecule was studied by Phillips (Phillips, 1997) while Lewis (Lewis et. al, 1972) provided a model to calculate the torsional energy barrier of the molecule. However, methyl group internal rotation draws a lot of attention from many scientific groups to explore its role in physical and chemical properties of molecules. The study of methyl torsion in methylated molecules is the area of interest from many years using experimental along with the theoretical investigation using ab initio calculations. Many scientific groups (Lister et. al, 1978;

$\square$ Corresponding Author E-Mail Address: swastisaxenaa@gmail.com 
Reed \& Weinhold, 1991; Payne \& Allen, 1977; Villard, 1974; Pitzer, 1983) have presented basic as well as deep reviews on this topic of interest. The steric repulsion, some electrostatic models, valence bond theory, hyperconjugation and some other concepts have been used to explain the origin of the torsional interactions. Srivastava et. al (Srivastava et. al, 2018) studied the effect of methyl group on different position of 2-hydroxypyridine molecule.

\section{Theoretical Background}

The geometrical optimization of the molecules was performed using ab initio calculations in the excited $\left(S_{1}\right)$ electronic state. The torsional barrier potential parameters have been obtained using the one dimension equation of torsional potential which is given by $\mathrm{V}(\tau)=(1 / 2) \mathrm{V}_{3}(1-\cos 3 \tau)$ $+(1 / 2) \mathrm{V}_{6}(1-\cos 6 \tau)$ where $\tau$ is torsional angle. All the ab initio calculations for excited electronic states were performed in Gaussian'09 (Frisch et. al, 2009) and the visualization of the molecular geometry and the molecular orbitals were carried out in Gabedit software (Allouche, 2011). The procedure used for the theoretical study of the molecules in their ground $\left(\mathrm{S}_{0}\right)$ and excited electronic state $\left(\mathrm{S}_{1}\right)$ has been discussed in details in our previous articles (Srivastava et. al, 2018; Sinha et. $\mathrm{al}, 2008)$.

\section{Experimental details}

The resonance enhanced multiphoton ionization (REMPI) experiment was performed using a tunable dye laser which was pumped by a second harmonic of the pulsed Nd:YAG laser (Litron nano series lasers, UK). For the ionization of the molecules, the output from the tunable dye laser was frequency doubled using another second harmonic crystal, while the scanning of the dye laser frequency was done by using stepper motor which was controlled by the developed LabVIEW program. The chemicals (4M2HP and 6M2HP) were purchased from alfa-aesar chemical company and used without further purification.

\section{Results and discussion}

\subsection{4-methyl-2-hydroxypyridine}

The REMPI spectrum of 4-methyl-2-hydroxypyridine in jet-cooled condition is shown in Fig. 1. The spectrum was recorded just after the release of the carrier gas. Many broad as well as sharp spectral bands were observed in the range of $34900 \mathrm{~cm}^{-1}$ to $35600 \mathrm{~cm}^{-1}$. The transition at 34987 $\mathrm{cm}^{-1}$ is assigned as the origin band of the molecule. Increasing the pressure of the carrier gas, does not provide any appreciable change in the intensity of the observed peak. Matsuda et. al (Matsuda et. al, 2001) showed the excitation spectrum of 2-hydroxypyridine in which the $\pi \pi^{*}$ transition band observed as the origin band. Thus, the origin band for 4-methyl-2-hydroxypyridine at $34987 \mathrm{~cm}^{-1}$ is assigned as $\pi \pi^{*}$ transition in a similar manner. $1136 \mathrm{~cm}^{-1}$ red shift observed for the origin band of 4-methyl-2-hydroxypyridine relative to the 2-hydroxypyridine molecule from the excitation spectrum. The plausible reason for this change could be the change in the electronic environment adjacent to the methyl group. In the obtained REMPI spectrum of 4M2HP, some other low frequency bands were observed near to origin band with less intensity. While in case of 2hydroxypyridine no such weak bands were reported near to origin band (Matsuda et. al, 2001). It may be due to the methyl internal rotational transitions. Though methyl torsional transitions show characteristic low frequency, presence of all these low frequency bands cannot be because of only 
methyl group internal motion. There could be the existence of $n \pi *$ transition state involving

Figure 1: REMPI spectrum of 4M2HP after 30 minutes of release of helium gas. The spectrum is normalized with the laser intensity.

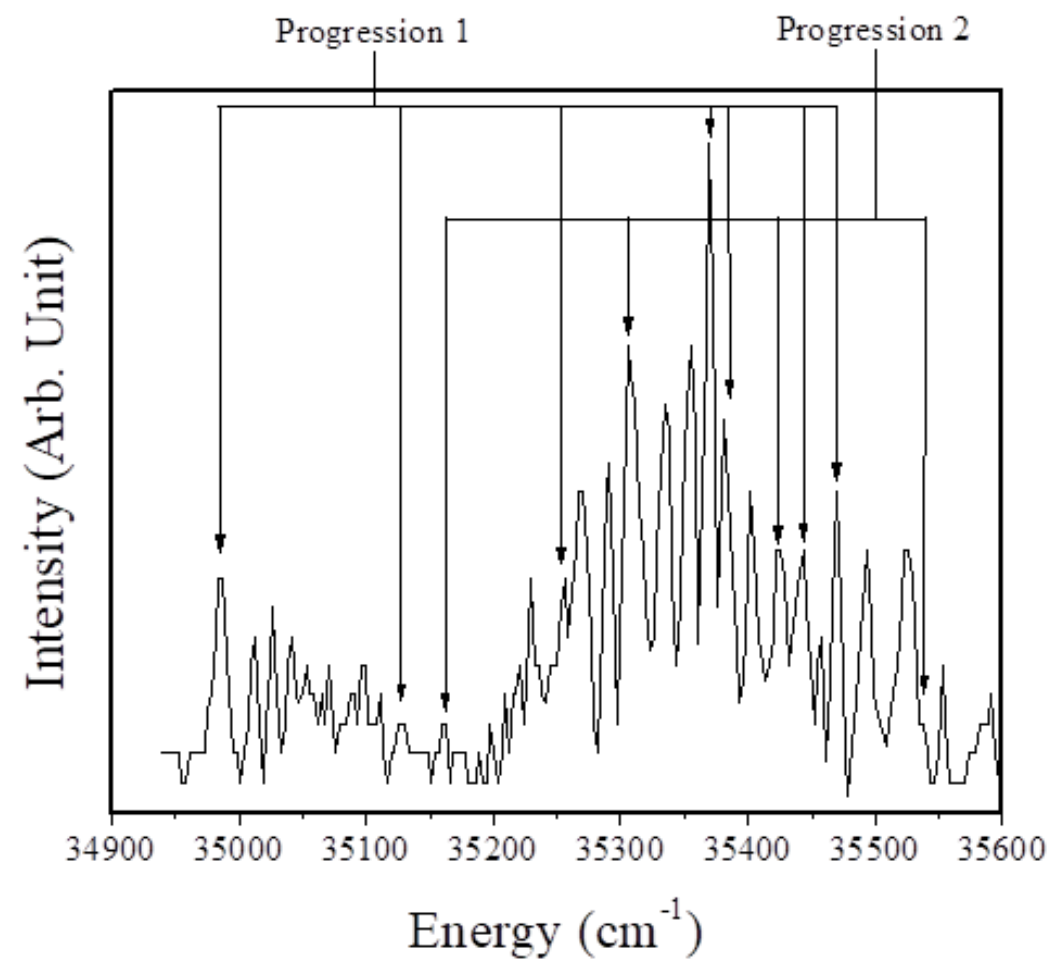

vibronic coupling with $\pi \pi^{*}$ transition state and because of that the low frequency vibration bands appear near to origin band.

At a separation of $26 \mathrm{~cm}^{-1}$, there observed a band with low intensity relative to the origin band. This band could be assigned as $2 \mathrm{e}$ torsional transition of methyl group which predicts the $3 \mathrm{a} 1$ and $4 \mathrm{e}$ torsional bands near to $57 \mathrm{~cm}^{-1}$ and $87 \mathrm{~cm}^{-1}$ respectively. Though there observed bands at 54 $\mathrm{cm}^{-1}$ and $83 \mathrm{~cm}^{-1}$ which can be assigned as $3 \mathrm{a} 1$ and $4 \mathrm{e}$ torsional bands, assignment of other bands will not be possible. Also, assigning $26 \mathrm{~cm}^{-1}$ as $2 \mathrm{e}$ band, produces a potential barrier of low magnitude. But the barrier potential in excited state was calculated with higher values of the potential parameters. Hence, this band at $26 \mathrm{~cm}^{-1}$ could also be assigned as some other band which associated with the $n \pi^{*}$ transition state. The $2 \mathrm{e}$ and $3 \mathrm{a} 1$ torsional transitions were assigned for the bands observed at $144 \mathrm{~cm}^{-1}$ and $269 \mathrm{~cm}^{-1}$ respectively. The potential parameters in the excited state were obtained by fitting these bands. The excited state torsional parameters $\left(\mathrm{V}^{\prime}{ }_{3}=536 \mathrm{~cm}^{-1} ; \mathrm{V}_{6}{ }_{6}\right.$ $=-10 \mathrm{~cm}^{-1} ; \mathrm{F}^{\prime}=5.3 \mathrm{~cm}^{-1}$ ) were obtained by the best fit of these observed bands. The bands observed at a separation of $383 \mathrm{~cm}^{-1}, 395 \mathrm{~cm}^{-1}, 459 \mathrm{~cm}^{-1}$ and $483 \mathrm{~cm}^{-1}$ from the origin band can be assigned as 5e, 6a2, 6a1 and 7e torsional transitions. The other observed bands were also assigned while the 4e band was not observed from the spectrum. While assigning the observed bands from the spectrum it was found that the band at $318 \mathrm{~cm}^{-1}$ could be observed due to the mixing of two vibrational modes. Thus the band at $173 \mathrm{~cm}^{-1}$ was assigned as the second lowest vibrational mode $\left(v^{1}\right)$ in the excited state and can explain the presence of band at $318 \mathrm{~cm}^{-1}$. The different vibrational bands and their assignments are tabulated in Tab. 1. 
There are many other vibrational modes observed in the spectrum due to the methyl torsional transitions which can be easily explained by the combination of the other vibrational modes as listed in Tab. 2 as progression 2. The bands obtained at $439 \mathrm{~cm}^{-1}$ and $551 \mathrm{~cm}^{-1}$ are assigned as $3 a 1$ and $5 \mathrm{e}$ and the torsion and vibration combination bands.

Table 1: Assignment of methyl group torsional transitions in REMPI spectrum of 4M2HP.

\begin{tabular}{|c|c|c|}
\hline Energy $\left.\mathbf{( c m}^{-\mathbf{1}}\right)$ & $\boldsymbol{\Delta v} \mathbf{( c m}^{-\mathbf{1}} \mathbf{)}$ & Assignment \\
\hline 34987 & 0 & Origin \\
\hline 35131 & 144 & $2 \mathrm{e}$ \\
\hline 35163 & 173 & $\boldsymbol{v}^{1}$ \\
\hline 35256 & 269 & $3 \mathrm{a} 1$ \\
\hline 35305 & 318 & $\boldsymbol{v}^{1}{ }_{0} 2 \mathrm{e}$ \\
\hline 35370 & 383 & $5 \mathrm{e}$ \\
\hline 35382 & 395 & $6 \mathrm{a} 2$ \\
\hline 35426 & 439 & $\boldsymbol{v}^{1}{ }_{0} 3 \mathrm{a} 1$ \\
\hline 35446 & 459 & $6 \mathrm{a} 1$ \\
\hline 35470 & 483 & $7 \mathrm{e}$ \\
\hline 35538 & 551 & $v^{1}{ }_{0} 5 \mathrm{e}$ \\
\hline
\end{tabular}

Table 2: Assignment of methyl group torsional transitions in REMPI spectrum of 4M2HP.

\begin{tabular}{|c|c|c|c|c|c|}
\hline \multicolumn{3}{|c|}{ Progression 1 } & \multicolumn{3}{c|}{ Progression 2 } \\
\hline$\left(\mathrm{S}_{0} \rightarrow \mathrm{S}_{1}\right)$ & Cal. $\left(\mathrm{cm}^{-1}\right)$ & Obs. $\left(\mathrm{cm}^{-1}\right)$ & $\left(\mathrm{S}_{0} \rightarrow \mathrm{S}_{1}\right)$ & Cal. $\left(\mathrm{cm}^{-1}\right)$ & Obs. $\left(\mathrm{cm}^{-1}\right)$ \\
\hline $1 \mathrm{e}-2 \mathrm{e}$ & 143 & 144 & $1 \mathrm{e}-2 \mathrm{e}$ & 143 & 145 \\
\hline $0 \mathrm{a} 1-3 \mathrm{a} 2$ & 143 & --- & $0 \mathrm{a} 1-3 \mathrm{a} 2$ & 143 & --- \\
\hline $0 \mathrm{a} 1-3 \mathrm{a} 1$ & 272 & 269 & $0 \mathrm{a} 1-3 \mathrm{a} 1$ & 270 & 266 \\
\hline $1 \mathrm{e}-4 \mathrm{e}$ & 274 & --- & $1 \mathrm{e}-4 \mathrm{e}$ & 272 & --- \\
\hline $1 \mathrm{e}-5 \mathrm{e}$ & 382 & 383 & $1 \mathrm{e}-5 \mathrm{e}$ & 378 & 378 \\
\hline $0 \mathrm{a} 1-6 \mathrm{a} 2$ & 394 & --- & $0 \mathrm{a} 1-6 \mathrm{a} 2$ & 391 & 393 \\
\hline $0 \mathrm{a} 1-6 \mathrm{a} 1$ & 455 & 439 & $0 \mathrm{a} 1-6 \mathrm{a} 1$ & 448 & --- \\
\hline $1 \mathrm{e}-7 \mathrm{e}$ & 488 & 483 & $1 \mathrm{e}-7 \mathrm{e}$ & 483 & --- \\
\hline
\end{tabular}

The change in the conformation of methyl group upon excitation from the ground state to excited state as observed in the excitation spectrum can be explained using the Frank-Condon factor calculation between these two states. This calculation of Frank-Condon factor reveals the information regarding the shift of the conformation of the methyl group while excitation. The observed REMPI spectrum of 4-methyl-2-hydroxypyridine suggests change in the conformation due to the internal rotation of methyl group upon excitation from ground electronic state $\left(S_{0}\right)$ to excited electronic state $\left(\mathrm{S}_{1}\right)$. The observed 5e transition band has been taken in account to estimate the torsional potential due to methyl group motion. B3LYP/TZVP level of theory has been used for further investigation of the conformational change and torsional potential in excited state $\left(\mathrm{S}_{1}\right)$. The optimized geometry of 4M2HP is shown in Fig. 2 (a). In minimum energy conformation of the molecule, the ring frame is always planar. The potential curve for the excited state $\left(\mathrm{S}_{1}\right)$ is shown in Fig. 2 (b). The calculated three fold potential term $\left(\mathrm{V}_{3}\right)$ was $517 \mathrm{~cm}^{-1}$. The obtained potential curve shows an internal rotation of methyl group upon excitation from ground state $\left(\mathrm{S}_{0}\right)$ to excited state $\left(\mathrm{S}_{1}\right)$. This is in agreement with the observed results from the optimized geometry of ground state and from the obtained REMPI spectrum. The change in methyl group conformation and magnitude of torsional potential barrier in 4M2HP upon excitation were explained using the $\pi^{*}-$ $\sigma^{*}$ hyperconjugation (Nakai \& Kawai, 2000). The $\pi^{*}-\sigma^{*}$ hyperconjugation in LUMO at the top of 
the barrier conformation stabilizes these in ground as well as in excited states. The orbital contour diagram for 4M2HP in HOMO and LUMO in minimum and top of the barrier conformation is shown in Fig. 3. $\pi^{*}-\sigma^{*}$ hyperconjugation was observed only in 180 degree conformation in LUMO. This signifies the change in conformation of methyl group in excited state i.e. rotation of methyl group upon excitation.

Figure 2: (a) The optimized geometry and (b) torsional angle dependence of potential barrier curve for $4 M 2 H P$ using B3LYP/TZVP level of theory.

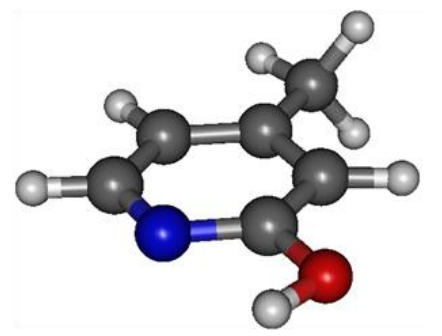

(a)

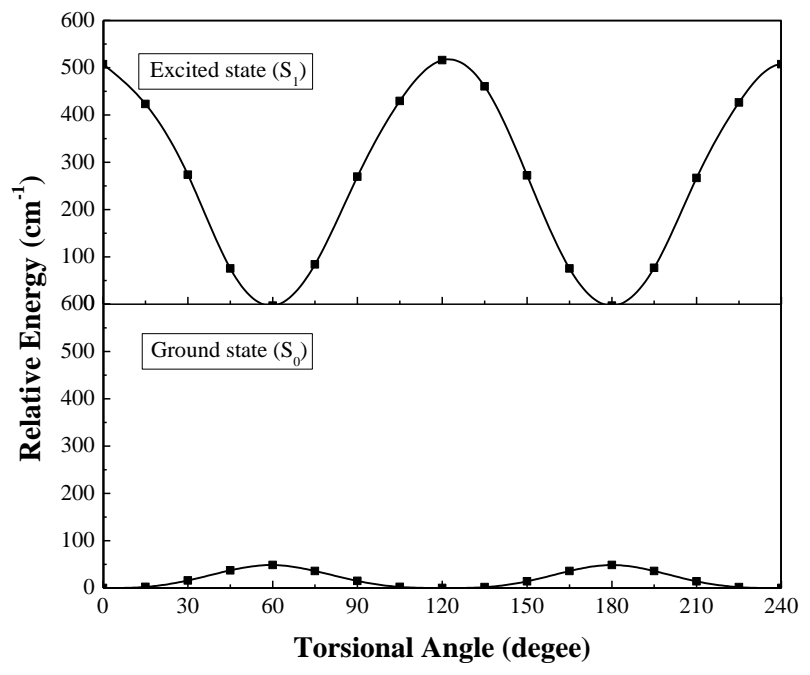

Figure 3: Contour diagrams of HOMO and LUMO of $4 \mathrm{M} 2 \mathrm{HP}$ in minimum energy conformation (0 degree) and top of the barrier conformation (180 degree). The contour diagrams are plotted with same sensitivity.
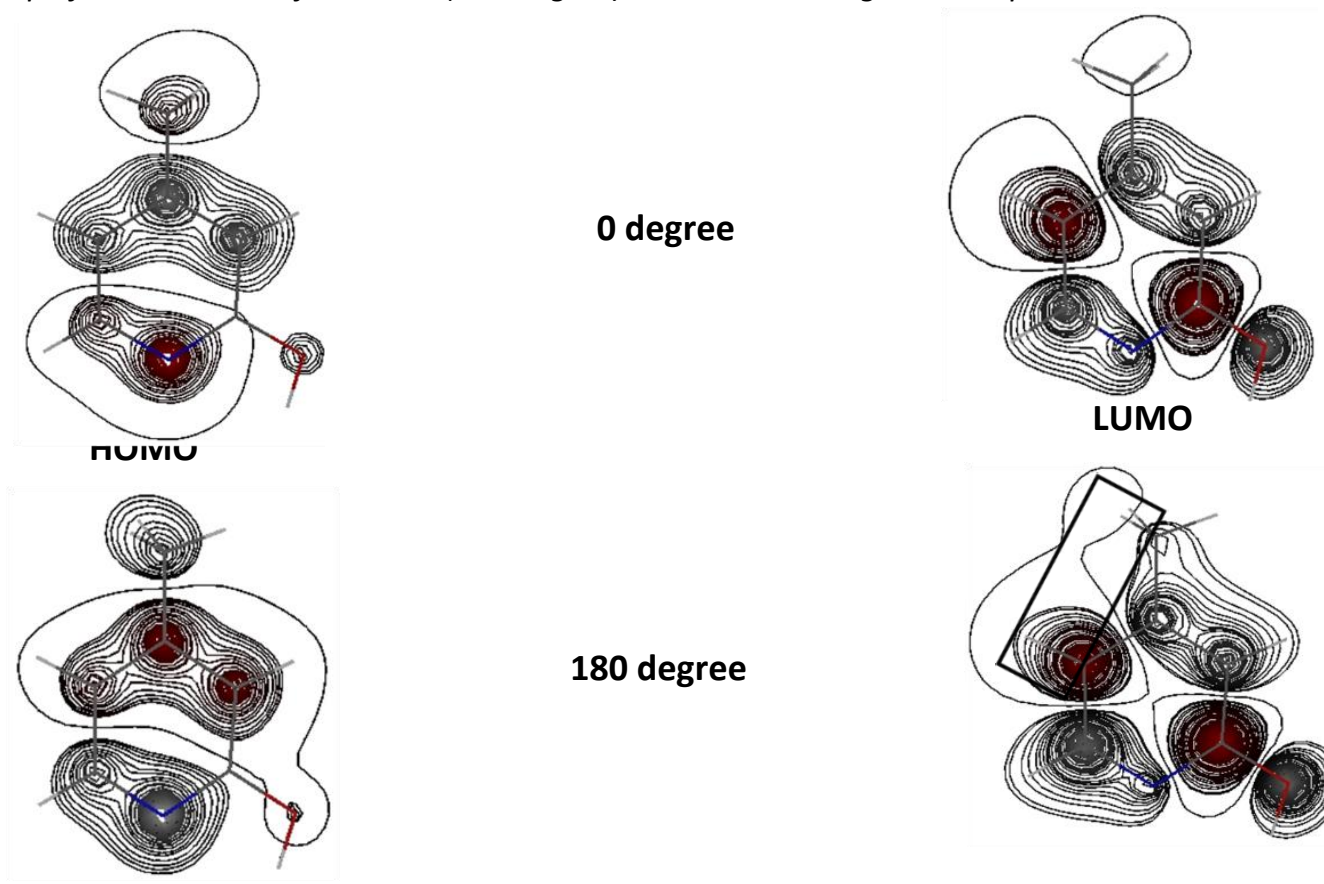

180 degree

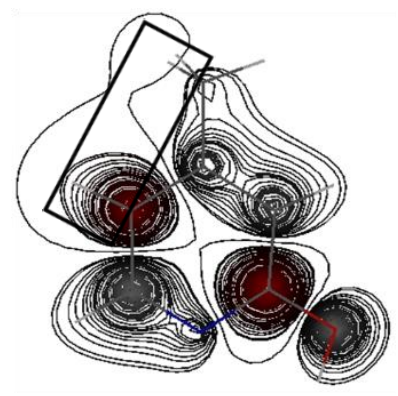

\subsection{6-methyl-2-hydroxypyridine}

The REMPI spectrum of jet-cooled 6-methyl-2-hydroxypyridine is shown in Fig. 4. The spectrum recorded after 30 minutes of release of the gas. One intense peak with few other peaks 
was observed in the spectral range of $35000 \mathrm{~cm}^{-1}$ to $35800 \mathrm{~cm}^{-1}$. The observed spectrum then normalized using the dye laser profile. The obtained transition band at $35405 \mathrm{~cm}^{-1}$ has been

Figure 4: REMPI spectrum of 6M2HP after 30 minutes of release of helium gas. The spectrum is normalized with

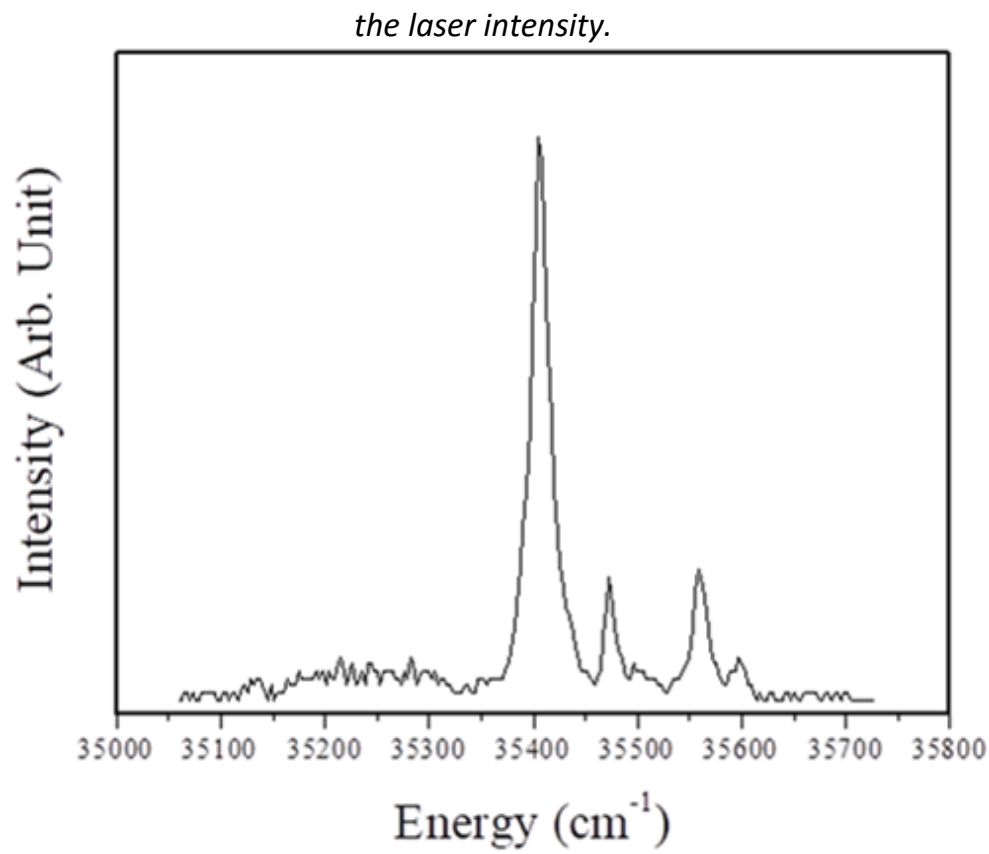

assigned as the origin band of the molecule. This assigned origin band is observed as $\pi \pi^{*}$ transition state. The characteristic of this band and the intensity pattern observed in the spectrum indicates unchanged molecular conformation in the excited state compared to the ground state.

The other bands obtained in the excitation spectrum of the molecule can be either vibrational bands or methyl torsional bands of the molecule. These bands are observed at a separation of 68, 153 and $192 \mathrm{~cm}^{-1}$ from the assigned origin band $\left(35405 \mathrm{~cm}^{-1}\right)$. The transition bands observed at 68 and $153 \mathrm{~cm}^{-1}$ were assigned as $3 \mathrm{a} 2$ and $3 \mathrm{a} 1$ transition bands with the experimentally obtained potential parameters $\mathrm{V}_{3}^{\prime}=265 \mathrm{~cm}^{-1} ; \mathrm{V}^{\prime}{ }_{6}=-84 \mathrm{~cm}^{-1}$ and $\mathrm{F}^{\prime}=5.3 \mathrm{~cm}^{-1}$. The other band observed at $192 \mathrm{~cm}^{-1}$ is assumed as some other vibration.

Table 3: Absolute and relative spectral positions of observed bands in the REMPI spectrum of 6M2HP.

\begin{tabular}{|c|c|c|}
\hline Energy $\left(\mathbf{c m}^{-\mathbf{1}}\right)$ & $\boldsymbol{\Delta v}\left(\mathbf{c m}^{-\mathbf{1}}\right)$ & Assignment \\
\hline 35405 & 0 & Origin \\
\hline 35473 & 68 & $3 \mathrm{a} 2$ \\
\hline 35558 & 153 & $3 \mathrm{a} 1$ \\
\hline 35597 & 192 & ---- \\
\hline
\end{tabular}

As observed from the obtained REMPI spectrum, there is no such conformational change due to the internal rotation of methyl group observed when excitation takes place from the ground electronic state $\left(S_{0}\right)$ to the excited electronic state $\left(S_{1}\right)$. B3LYP/TZVP level of theory have been taken in account for the further investigation of the conformation and torsional potential of the molecule in excited state $\left(\mathrm{S}_{1}\right)$. The optimized geometry of $6 \mathrm{M} 2 \mathrm{HP}$ in excited state is shown in Fig. 5 (a). The ring frame is always planar whenever geometry optimization in minimum energy conformation has been performed. The calculated intensity pattern, using the obtained potential parameters for ground as well as for excited state from the theoretical study, is in match with the result obtained from the excitation spectrum. Hence, there is no change in conformation of methyl 
group observed. The torsional angle dependence of the potential energy curve for the excited state $\left(\mathrm{S}_{1}\right)$ is shown in Fig. 5 (b).

Figure 5: (a) The optimized geometry and (b) torsional angle dependence of potential barrier curve for $6 M 2 H P$ using B BLYP/TZVP level of theory.

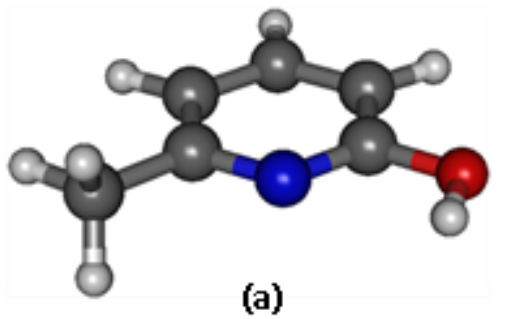

(b)

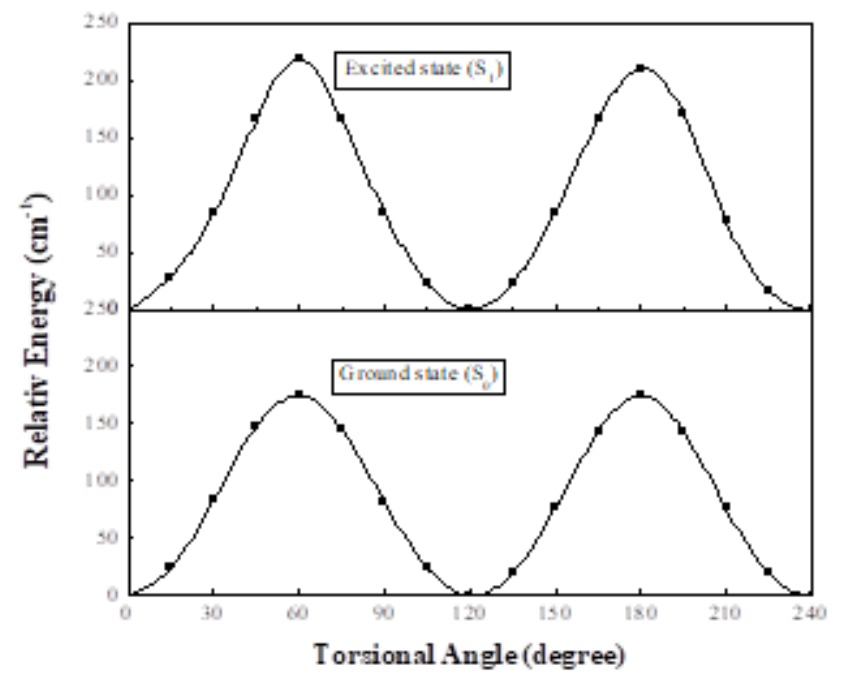


The calculated three-fold potential term $\mathrm{V}_{3}$ was found to be $212 \mathrm{~cm}^{-1}$. The obtained potential curve for the optimized geometry is in agreement with the observed REMPI spectrum of the molecule. There is no change of methyl group conformation observed in REMPI spectrum, hence no $\pi^{*}-\sigma^{*}$ hyperconjugation involves. The orbital contour diagram for 6M2HP in HOMO and LUMO in minimum and top of the barrier conformation is shown in Fig. 6.

Figure 6: Contour diagrams of HOMO and LUMO of 6M2HP in minimum energy conformation (0 degree) and top of the barrier conformation (180 dearee). The contour diagrams are plotted with same sensitivity.

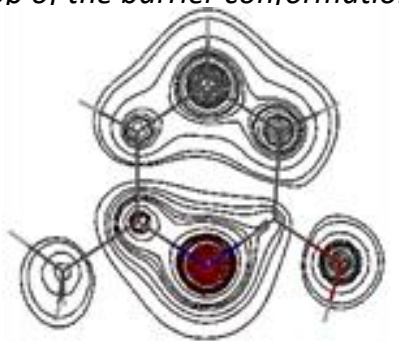

HOMO

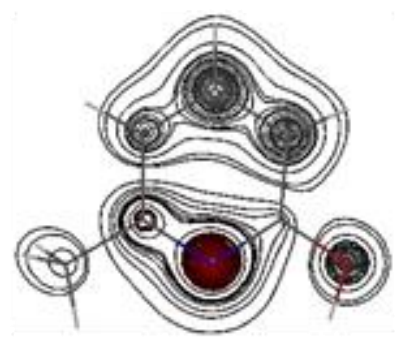

0 degree

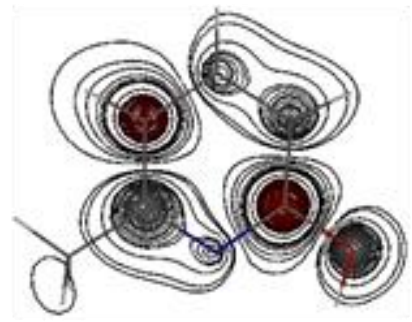

LUMO

180 deoree

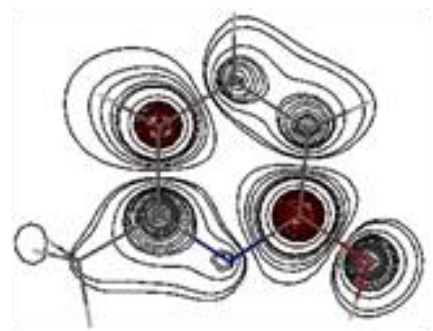

\section{Conclusion}

The ab initio calculation as well as the intensity pattern shows the conformational change in the methyl group in 4M2HP upon excitation from ground state to excited state. The band observed at 34987 and $35405 \mathrm{~cm}^{-1}$ was assigned as $\pi \pi^{*}$ transition state and named as origin band for 4M2HP and 6M2HP respectively. Many low frequency vibrational bands were observed near the origin band in 4M2HP which could be because of the vibronic coupling of the $n \pi^{*}$ and $\pi \pi^{*}$ transition states. The $\pi^{*}-\sigma^{*}$ hyperconjugation is responsible term for the conformational change in methyl group in $4 \mathrm{M} 2 \mathrm{HP}$ while no hyperconjugation observed in 6M2HP.

\section{Acknowledgment}

The authors would like to thank the Department of Science and Technology, India for financial support. 


\section{References}

[1] Suzuki H. (1959). Relations between electronic absorption spectra and spatial configurations of conjugated systems (I). Biphenyl. Bull. Chem. Soc. Jpn., 32(12), 1340-1350.

[2] Hargreaves A. and Rizvi S. H., (1962). The crystal and molecular structure of biphenyl, Acta Crystallographica, 15(4):365-373.

[3] Im H. and Bernstein E. R., (1988) Geometry and torsional motion of biphenyl in the ground and first excited singlet state. J. Chem. Phys., 88(12), 7337-7347.

[4] Phillips D., (1997) Studies on torsional motion in aromatic amines. J. Photochem. Photobiol. A: Chem., 105(2-3), 307-315.

[5] Lewis J. D., Malloy Jr. T. B., Chao T. H., and Laane J., (1972). Periodic potential functions for pseudo rotation and internal rotation. J. Mol. Struct., 12(3), 427-449.

[6] Lister D. G., McDonald J. N., and Owen L. N., (1978), Internal Rotation and Inversions: An introduction to Large Amplitude Motions in Molecules, Academic Press Inc., New York.

[7] Reed A. E. and Weinhold F., (1991). Natural bond orbital analysis of internal rotation barriers and related phenomena. Isr. J. Chem., 31(4), 277-285.

[8] Payne P. W. and Allen L. C., (1977) Barriers to Rotation and Inversion, Springer US, Boston, MA.

[9] Villard A. (1974). Internal Rotation in Molecules. John Wiley and Sons, New York.

[10] Pitzer R. M. (1983). The barrier to internal rotation in ethane. Acc. Chem. Res., 16(6), 207210.

[11] Srivastava A. K., Sinha R. K., Saxena S. and Kundu T. (2018). Int. J. Chem. Sci., 16(2): 270 (1-13). DOI: $10.21767 / 0972-768 X .1000270$

[12] Frisch M. J., Trucks G.W., Schlegel H. B., Scuseria G. E., and Robb M. A. et. al. (2009). Gaussian'09 Revision E.01. Gaussian Inc. Wallingford CT.

[13] Allouche A. R., (2011). Gabedit "A graphical user interface for computational chemistry softwares". J. Comp. Chem., 32(1), 174-182.

[14] Sinha R. K., Singh B. P., and Kundu T., (2008). Theo. Chem. Acc., 121(1), 59-70.

[15] Matsuda Y., Ebata T. and Mikami N., (2001). IR-UV double resonance spectroscopic study of 2-hydroxypyridine and its hydrogen-bonded clusters in supersonic jets, J. Phys. Chem. A 105(14), 3475-3480.

[16] Nakai H. and Kawai M. (2000). Hyperconjugation mechanism on the rotational barrier of the methyl group (i): Substituted toluenes in the ground, excited, and anionic states, J. Chem. Phys., 113(6):2168-2174. 